\title{
Effect of Different Heat Treatment on Alkylresorcinol Contents of Wheat Bran
}

\begin{abstract}
Shuangqi Tian, Renyong Zhao,* Tianyuan Peng, Chenxi Liu, and Yingqi Yang
The influence of different heat treatment levels on the contents of alkylresorcinol and homologues (ARs) was evaluated using wheat bran from two different wheat cultivars. The ARs in the wheat bran were destroyed by all heat treatments investigated in this study (oven treatment, microwave treatment, autoclave treatment, and extrusion treatment). The results showed that the loss rate of ARs by different heat treatment was oven treatment $>$ autoclave treatment $>$ extrusion treatment $>$ microwave treatment, indicating that microwave treatment was more suitable for stabilizing wheat bran than the other three heat treatments. Both temperature and time of heat treatment had effects on the ARs of wheat bran. Higher temperatures and longer times of the treatment resulted in higher loss rates of ARs of wheat bran. The thermal stability of ARs homologues was different between wheat varieties, which may result from the variance of their structure and other components in wheat bran.
\end{abstract}

Keywords: Wheat bran; Alkylresorcinol; ARs; Heat treatment

Contact information: College of Food Science and Technology, Henan University of Technology, Zhengzhou 450001, China; *Corresponding author: zry8600@126.com

\section{INTRODUCTION}

Lipase and lipoxygenase in wheat bran can catalyze lipid hydrolysis or oxidation rancidity during storage, which reduces the storage stability of wheat bran. These changes can affect the function, organoleptic, and nutrition characteristics of wheat bran, which limits its application (Srivastava et al. 2007; Sudha et al. 2007; Rikard et al. 2008). Heat inactivation of the enzymes in wheat bran and germ is an effective way to improve the storage stability of whole wheat flour (Rose et al. 2008; Dobladomaldonado et al. 2012). Generally, whole wheat flours are prepared by combining the bran and germ with fine flour (Podio et al. 2019). Stabilization treatment of bran and germ before combining can improve the storage stability of whole wheat flour (Li et al. 2007; Jacobs et al. 2016).

Heat treatment results in the loss of some heat-sensitive nutrients, such as phenols, anthocyanins, and alkylresorcinol and homologues (ARs) (Randhir et al. 2008; Demir and Elgün 2014; Wang et al. 2019). ARs are important phenolic component in whole wheat flour, and the ARs content is a quality evaluation index of whole wheat flour (Kozubek and Tyman 1999; AI-Ruqaie, and Lorenz 1992). Some potential health effects, such as anticancer, antioxidants, antimicrobial, and antimutagenic, are related to the intake of ARs in animal and model systems (Landberg et al. 2006). However, there has been less research about the effect of heat treatment on the ARs in wheat bran. In the process of different heat treatments, to reduce the loss of ARs and improve the quality of wheat bran, the change of ARs content was studied. Because ARs are absent in the endosperm and exclusively deposited in the outer layers of wheat, wheat bran is used instead of whole wheat flour (Bordiga et al. 2016; Majewski, and Cunha 2018). 
The present study investigated the variation of ARs content and homologues compositions in wheat bran by different heat treatments using various cultivars of two different wheat brans. The wheat brans of two different types were heated by physical treatment methods such as oven, microwave, autoclave, and extrusion. The ARs and homologues in bran were collected by ultrasound-assisted extraction.

\section{EXPERIMENTAL}

\section{Materials and Chemicals}

Bran 1 was provided by Cofco Zhengzhou Haijia Food Co. Ltd. (Zhengzhou, China), and bran 2, both untreated and extruded forms, was provided by Hebei Black Horse Flour Co. Ltd. (Shijiazhuang, China). The contents of each component in wheat bran are listed in Table 1. Ethyl acetate and acetic acid were analytical grade and purchased from Tianjin HengXing Chemical Reagent Co. Ltd. (Tianjin, China). Methanol (chromatographic grade), Fast Blue B Zn, and the authentic standards 5pentadecylresorcinol (C15:0) were obtained from Sigma-Aldrich (Saint Louis, USA). Nitrogen was purchased from Zhengzhou Zezhong Technology Co. Ltd. (Zhengzhou, China).

\section{Heat Treatments for Wheat Bran}

Oven drying

A total of $60 \mathrm{~g}$ of each bran was placed on china plates $(20 \mathrm{~cm}$ in diameter) to achieve a height of $2.5 \mathrm{~cm}$ and subjected to convective oven for $30 \mathrm{~min}$ at $90{ }^{\circ} \mathrm{C}, 110{ }^{\circ} \mathrm{C}$, $130{ }^{\circ} \mathrm{C}, 150{ }^{\circ} \mathrm{C}$, and $170^{\circ} \mathrm{C}$. A total of $60 \mathrm{~g}$ of each bran was placed on china plates and heated in an oven at $150{ }^{\circ} \mathrm{C}$ and $170{ }^{\circ} \mathrm{C}$ for $5 \mathrm{~min}, 10 \mathrm{~min}, 15 \mathrm{~min}, 20 \mathrm{~min}, 25 \mathrm{~min}$, or 30 $\min$.

\section{Microwave treatment}

A total of $150 \mathrm{~g}$ of each bran was placed on china plates to achieve a height of 2.5 $\mathrm{cm}$. Bran specimens under the same conditions (microwave irradiation power $700 \mathrm{~W}$, oscillation frequency of $2450 \mathrm{~Hz}$ ) were treated for $30 \mathrm{~s}, 90 \mathrm{~s}, 150 \mathrm{~s}, 210 \mathrm{~s}$, or $270 \mathrm{~s}$, and the temperature of treated brans was measured.

\section{Autoclave treatment}

A total of $150 \mathrm{~g}$ of each bran was placed on china plates to achieve a height of 2.5 $\mathrm{cm}$. Brans were steamed at the different temperatures $\left(110^{\circ} \mathrm{C}, 120^{\circ} \mathrm{C}, 130{ }^{\circ} \mathrm{C}\right)$ for $5 \mathrm{~min}$, $10 \mathrm{~min}, 15 \mathrm{~min}, 20 \mathrm{~min}$, or $25 \mathrm{~min}$.

\section{Extrusion treatment}

A DS32- II twin-screw extrusion tester (Saixin Puffing Machinery Co., Ltd. Jinan, China) was used for experiments. The temperature in zone I was $40{ }^{\circ} \mathrm{C}$, zone II $140{ }^{\circ} \mathrm{C}$, and zone III $160{ }^{\circ} \mathrm{C}$. The prepared bran 2 were extruded under the condition of main machine frequency $15 \mathrm{~Hz}$, feeding frequency $10 \mathrm{~Hz}$, and rotary cutting frequency $60 \mathrm{~Hz}$. 


\section{Alkylresorcinol Analyses}

Extraction of ARs in bran

Ultrasound-assisted extraction of ARs was performed using a Scientz-IID ultrasonic cell disruptor (Ningbo, China), containing a $\Phi 6$ amplitude transformer. An aliquot of $0.8 \mathrm{~g}$ of whole wheat flour and $40 \mathrm{~mL}$ of ethyl acetate were sonicated for $2 \mathrm{~min}$ at $286 \mathrm{~W}$ (turn ultrasonic cell disruptor for one second, the effective ultrasonic time within $1 \mathrm{~min}$ is $30 \mathrm{~s}$ ). To avoid the loss of solvent during heating, samples were cooled in an ice bath during ultrasonication. The extract was centrifuged for $10 \mathrm{~min}$ at $3000 \mathrm{r} / \mathrm{min}$. After $5 \mathrm{~mL}$ of obtained suspension was evaporation by nitrogen, the dried extract was dissolved in $1 \mathrm{~mL}$ of methanol and filtered through a $0.22 \mu \mathrm{m}$ membrane. All extract solutions were freshly prepared daily prior to use.

\section{Determination of ARs in bran}

A stock solution of $0.05 \%(w / v)$ Fast Blue B salt was prepared in methanol containing $1 \%(\mathrm{v} / \mathrm{v})$ acetic acid (Gajda et al. 2008). A fresh working solution of Fast Blue B reagent was prepared by mixing 1 part stock reagent with 5 parts methanol. Stock solutions of pure olivetol were prepared in methanol at a concentration of $1 \mathrm{mg} / \mathrm{mL}$. Aliquots of this solution between 1 and $10 \mu \mathrm{L}$ ( 1 to $10 \mu \mathrm{L}$, or 6.3 to $62.9 \mathrm{nmol}$ ) were placed in assay tubes and made up to $200 \mu \mathrm{L}$ with methanol. Then, $2 \mathrm{~mL}$ of working solution of Fast Blue B salt was added to each assay tube. Samples extracted from cereal grains were also assayed. The absorbance was measured after $60 \mathrm{~min}$ at $520 \mathrm{~nm}$. Each experiment was done in triplicate.

\section{Statistical Analysis}

The results were expressed as mean \pm standard deviation (SD) of at least three independent experiments. Using SPSS 16.0 software (SPSS Inc., Chicago, IL, USA) for variance analysis, the Duncan test was selected to carry out significant and statistical analysis of data at the $\mathrm{P}<0.05$ level. Origin 9.0 software (Northampton, USA) was used for data visualization.

\section{RESULTS AND DISCUSSION}

\section{Effect of Oven Treatment on ARs Contents}

To ensure product quality, wheat bran must be stabilized before milling. Therefore, the effect of heat treatment on two kinds of bran ARs can reflect the ARs in different varieties of wheat bran. The results also reflect the effect of heat treatment on the ARs in different varieties of wheat bran.

Table 1. Contents of each Component in Wheat Bran (\%)

\begin{tabular}{|c|c|c|c|c|c|}
\hline Variety & Protein & Fat & Starch & Ash & Total dietary fibre \\
\hline Bran1 & $17.66 \pm 0.38$ & $4.16 \pm 0.03$ & $7.90 \pm 0.50$ & $17.58 \pm 0.91$ & $45.31 \pm 1.12$ \\
\hline Bran 2 & $17.81 \pm 0.09$ & $2.89 \pm 0.01$ & $14.03 \pm 0.84$ & $24.74 \pm 0.37$ & $38.09 \pm 0.87$ \\
\hline
\end{tabular}

Oven treatment had a strong influence on ARs content in wheat bran. The change of total ARs content in the bran is shown in Fig. 1. The oven treatment damaged the ARs. As the temperature of the oven increased, the total content of ARs in bran gradually 
decreased, while the loss rate of ARs gradually increased. Initially, the ARs content of two different brans were $1802.7 \pm 7.4 \mu \mathrm{g} / \mathrm{g}$ and $1615.3 \pm 5.0 \mu \mathrm{g} / \mathrm{g}$. When the temperature was raised to $110{ }^{\circ} \mathrm{C}$, the content of ARs dropped significantly to $1723.2 \pm 31.8 \mu \mathrm{g} / \mathrm{g}$ and $1481.8 \pm 25.5 \mu \mathrm{g} / \mathrm{g}(\mathrm{P}<0.05)$. After the temperature rise to $130{ }^{\circ} \mathrm{C}$, the loss rate of ARs kept increasing, and the content of ARs in bran were significantly different when comparing the total content $(\mathrm{P}<0.05)$. When the heat temperature was $170{ }^{\circ} \mathrm{C}$, the total content of ARs in the two kinds of bran decreased to $1275.3 \pm 0.0 \mu \mathrm{g} / \mathrm{g}$ and $953.8 \pm 0.0$ $\mu \mathrm{g} / \mathrm{g}$.

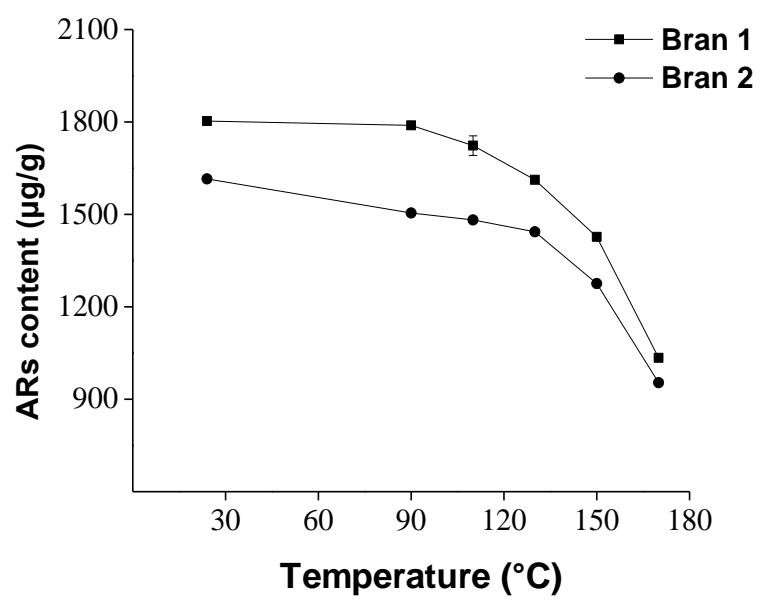

Fig. 1. Effect of oven treatment temperature on ARs content of wheat bran

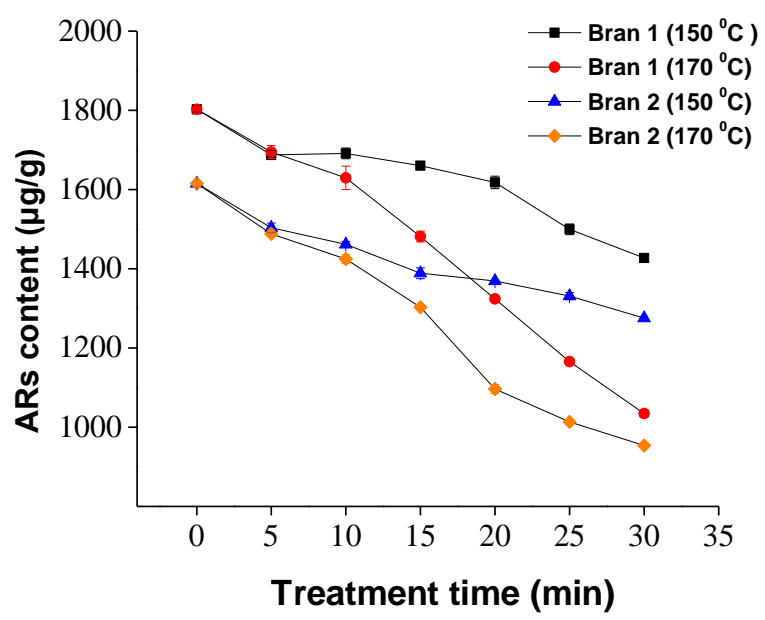

Fig. 2. Effect of oven treatment time on ARs content of wheat bran

To elucidate the relationship between ARs total content and heating time, two kinds of brans were treated at $150{ }^{\circ} \mathrm{C}$ and $170{ }^{\circ} \mathrm{C}$ for $5 \mathrm{~min}, 10 \mathrm{~min}, 15 \mathrm{~min}, 20 \mathrm{~min}, 25$ min, and 30 min. The ARs total content in bran is shown in Fig. 2.

Figure 2 shows that ARs total contents in bran 1 and bran 2 were significantly decreased after $5 \mathrm{~min}$ at $150{ }^{\circ} \mathrm{C}$ and $170{ }^{\circ} \mathrm{C}$ in the oven $(\mathrm{P}<0.05)$. The ARs total content in bran 1 and bran 2 were $1802.7 \pm 7.4 \mu \mathrm{g} / \mathrm{g}$ and $1615.3 \pm 5.0 \mu \mathrm{g} / \mathrm{g}$, while heated bran1 and bran 2 at $150{ }^{\circ} \mathrm{C}$ contained $1687.4 \pm 11.7 \mu \mathrm{g} / \mathrm{g}$ and $1503.6 \pm 11.7 \mu \mathrm{g} / \mathrm{g}$. When bran 1 and bran 2 were heated at $170{ }^{\circ} \mathrm{C}$ for $5 \mathrm{~min}$, the ARs total content was reduced to 1694.8 
$\pm 16.3 \mu \mathrm{g} / \mathrm{g}$ and $1488.1 \pm 2.3 \mu \mathrm{g} / \mathrm{g}$, respectively. Heating time had a significant effect on the total content of ARs in the bran within 0 to $30 \mathrm{~min}(\mathrm{P}<0.05)$, which showed a linear reduction trend. The ARs total content and heating time in bran 1 at $150{ }^{\circ} \mathrm{C}$ was as follows,

ARs $=-11.25 \times$ treatment time $+1795.4\left(\mathrm{R}^{2}=0.9188\right)$

The ARs total content and heating time in bran 1 at $170{ }^{\circ} \mathrm{C}$ was as follows,

ARs $=-26.21 \times$ treatment time $+1840.6\left(\mathrm{R}^{2}=0.9856\right)$

The ARs total content and heating time in bran 2 at $150{ }^{\circ} \mathrm{C}$ was as follows,

ARs $=-10.413 \times$ treatment time $+1576.9\left(\mathrm{R}^{2}=0.9543\right)$

The ARs total content and heating time in bran 2 at $170{ }^{\circ} \mathrm{C}$ was as follows,

$$
\text { ARs }=-23.299 \times \text { treatment time }+1620.1\left(\mathrm{R}^{2}=0.9798\right)
$$

Oven treatment in certain conditions destroyed the ARs in wheat bran. This result was similar to the impact of heat treatment on ARs total content in mango peel (Christian et al. 2015). During the drying process, the ARs were more stable at lower temperature. As the treatment temperature was increased, the stability and the total content of ARs decreased. The extension of treatment time could accelerate the loss of ARs. The reduced ARs contents of brans after convective oven heating might be due to aeration. The circulating gas in the oven provided sufficient oxygen for the oxidation reaction of ARs. In addition, the thermal ARs degradation during oven heating might also contribute to ARs losses (Randhir et al. 2008; Geerkens et al. 2015). Therefore, it should avoid high temperature and long treatment time to reduce the loss of ARs in the process of oven treatment.

\section{Effect of Microwave Treatment on ARs Contents}

Microwave processing has the characteristics of time-saving, high efficiency, and less nutrition loss, but it results in changes in the quality of lipids. Because ARs are commonly phenolic lipids, microwave treatment may cause the content to change (Vadivambal and Jayas 2007). To study the effect of microwave treatment on ARs in wheat bran, the brans were treated by microwave for $30 \mathrm{~s}, 90 \mathrm{~s}, 150 \mathrm{~s}, 210 \mathrm{~s}$, and $270 \mathrm{~s}$. The ARs total content and temperature of bran 1 and bran 2 are shown in Fig. 3.

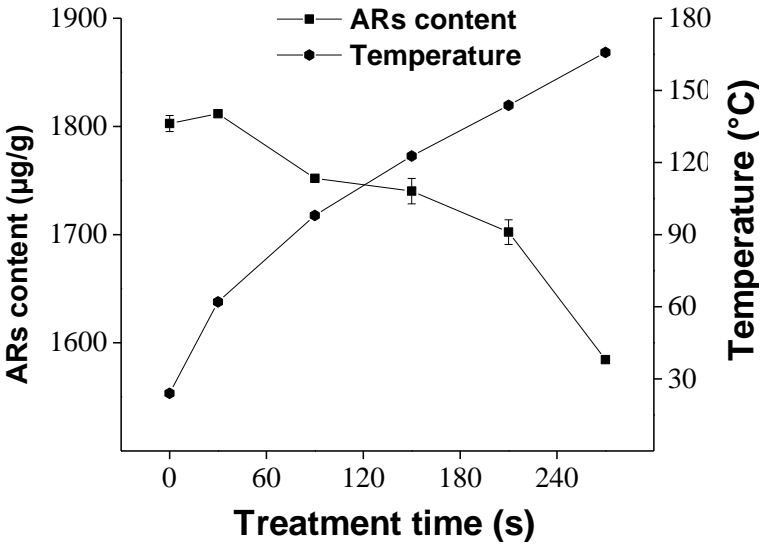

A

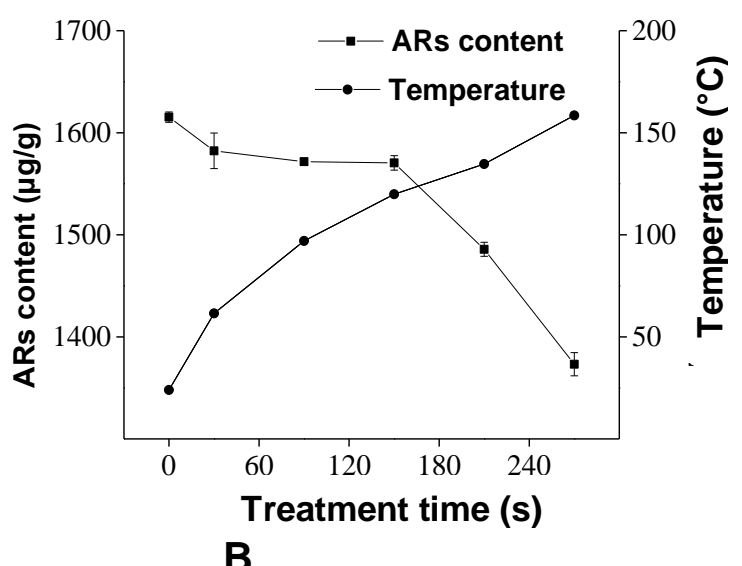

B

Fig. 3. Effect of microwave treatment on ARs content of wheat bran (A: bran1; B: bran 2) 
As shown in Fig. 3, the bran temperature increased and the total content of ARs in the bran decreased gradually with the increase of microwave treatment time. After two kinds of bran were treated by microwave for $270 \mathrm{~s}$, the loss rate of ARs was $12.1 \pm 0.1 \%$ and $15.0 \pm 0.7 \%$, and the bran temperature were as high as $165.8{ }^{\circ} \mathrm{C}$ and $165.8{ }^{\circ} \mathrm{C}$, respectively.

These results showed that the longtime microwave treatment reduced the ARs total content in wheat bran. Because the bran temperature was increased by microwave treatment, the reduced ARs content might be due to the oxidation, thermal degradation, or polymerization of ARs during processing (Geerkens et al. 2015). It may also relate to the non-thermal effect of microwave, such as magnetic field effect. Microwave induces the production of free radicals, which can lead to different chemical reactions and cause changes in oil quality (Hoogenboom 2009). The ARs of bran is likely to be changed in the process of scavenging free radicals. Therefore, long-time microwave heating of bran should be avoided to reduce damage in ARs.

\section{Effect of Autoclave Treatment on ARs Contents}

The changes of ARs total content in the bran during autoclave treatment are shown in Fig. 4. Under the different temperatures, the total ARs contents in two kinds of brans decreased gradually with the extension of treatment time. The loss rate of ARs in two kinds of bran were similar and less affected by the initial content. When the temperature was set to $110{ }^{\circ} \mathrm{C}, 120{ }^{\circ} \mathrm{C}$, and $130{ }^{\circ} \mathrm{C}$, the ARs total content in bran were significantly lower than the control after $5 \mathrm{~min}(\mathrm{P}<0.05)$. After $10 \mathrm{~min}$, the total contents of ARs in bran tended to be steady as the treatment time increased further. In autoclave treatment process, brans had a high water content, and water was a good heat transfer medium, which promotes the catalytic reaction of ARs by enzymes and accelerated the damage of ARs by heat treatment. With the further increase of brans temperature, the enzyme activity decreased, and the change of the total ARs content was steady. In addition, the total content of ARs in the bran was affected by the treatment temperature. At the treatment condition of $130{ }^{\circ} \mathrm{C}$ and $25 \mathrm{~min}$, the ARs loss rate of the two kinds of bran were $27.3 \pm$ $0.1 \%$ and $34.1 \pm 0.8 \%$.

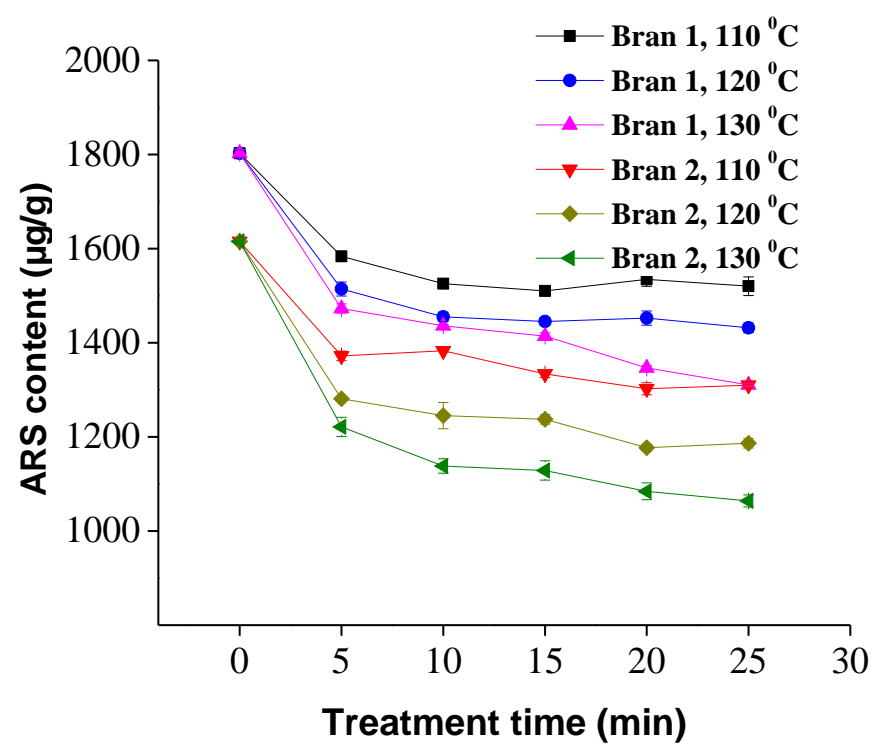

Fig. 4. Effect of autoclaving on ARs content of wheat bran 
According to the above results, autoclave treatment can destroy ARs in wheat bran, resulting in a decrease in its content, and this change mainly occurs in the first 10 min. The total content of ARs was affected by both heating temperature and time. A higher temperature resulted in a lower total amount of ARs. Under the same temperature, a longer processing time resulted in a lower total content of ARs, until it tends to be stable.

\section{Comparison of the Effects of Different Heat Treatment on ARs Contents}

Figure 5 compares the total amount of the ARs after oven processing (under the condition of $170{ }^{\circ} \mathrm{C}$ for $30 \mathrm{~min}$ ), microwave treatment $(270 \mathrm{~s}$ ), autoclave treatment (under the condition of $130{ }^{\circ} \mathrm{C} 25 \mathrm{~min}$ ), and bran extrusion processing.

After the four kinds of heat treatment, the total ARs content of both bran decreased significantly $(\mathrm{P}<0.05)$, suggesting that the oven treatment, microwave treatment, autoclave treatment, and extrusion treatment damage the ARs in bran (Fig. 5). Under the same heating conditions, two brans displayed a small difference in the ARs loss rate, which may be due to the difference in the composition and structure of ARs homologues in different bran (Gunenc et al. 2013). In addition, some material in the bran, such as lipase or antioxidant activity components, may have impacted the ARs during the treatment and cause differences in ARs loss rate in different bran varieties.

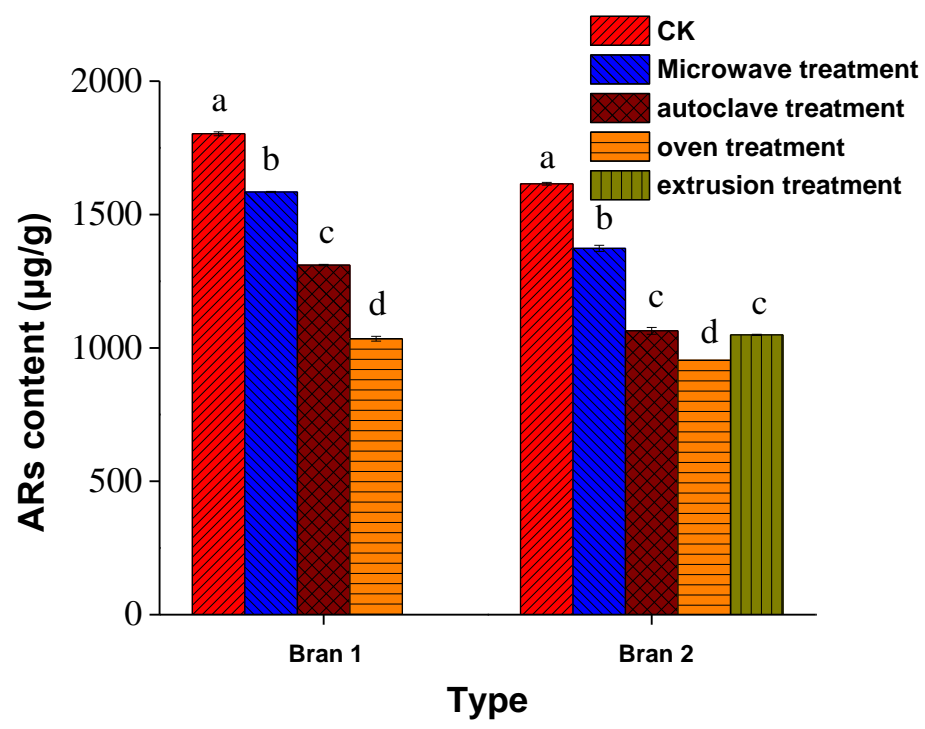

Fig. 5. Effect of heat treatments on ARs content of bran from different wheat varieties. The bran treated by different treatment methods is marked with different lowercase letters, indicating that the difference between different treatment methods is significant $(P<0.05)$.

The variation in ARs damage induced by different heat treatments may be related to the effect of different heating patterns. During oven processing, the bran is mainly affected by high temperature. In autoclave treatment, the bran is subjected to high temperature and high pressure, and the moisture content in the sample is high. During extrusion, the bran is subjected to high temperature, high pressure, and shear force (Rose et al. 2008; Oladiran et al. 2018). For both bran types, oven treatment led to the biggest reduction in ARs content, followed by autoclave and microwave. Therefore, microwave treatment is more suitable for stabilizing wheat bran due to less ARs loss. 


\section{CONCLUSIONS}

1. Oven treatment, microwave treatment, autoclave treatment, and extrusion treatment have destructive effects on ARs in wheat bran to different degrees. The effect of heat treatments on ARs was oven treatment > autoclave treatment, extrusion treatment > microwave treatment, indicating that microwave treatment was more suitable for stabilizing wheat bran than the other three heat treatments.

2. The ARs were affected by heat treatment time and temperature. A higher temperature and longer processing time resulted in higher loss rates of ARs.

3. The effect of heat treatment on ARs in different varieties of wheat bran was different, which may be caused by the differences in the composition and structure of ARs homologues and the influence of other substances in the bran.

\section{ACKNOWLEDGEMENTS}

The authors would like to acknowledge for financial assistance under National Key R and D Program of China (2018YFD0401000) and NSFC Research Contract no. 31701636.

\section{CONFLICT of INTEREST}

The authors declare that there is no conflict of interest.

\section{REFERENCES CITED}

AI-Ruqaie, I., and Lorenz, K. (1992). "Alkylresorcinols in extruded cereal brans," Cereal Chemistry 69(5), 472-475.

Bordiga, M., Locatelli, M., Travaglia, F., Arlorio, M., Reyneri, A., Blandino, M., and Coisson, J. D. (2016). "Alkylresorcinol content in whole grains and pearled fractions of wheat and barley," Journal of Cereal Science 70, 38-46. DOI: 10.1016/j.jcs.2016.05.017

Demir, M. K., and Elgün, A. (2014). "Comparison of autoclave, microwave, IR and UVC stabilization of whole wheat flour branny fractions upon the nutritional properties of whole wheat bread," Journal of Food Science and Technology 51(1), 59-66. DOI: 10.1007/s13197-011-0475-0

Dobladomaldonado, A. F., Pike, O. A., Sweley, J. C., and Rose, D. J. (2012). "Key issues and challenges in whole wheat flour milling and storage," Journal of Cereal Science 56(2), 119-126. DOI:10.1016/j.jcs.2012.02.015

Gajda, A., Kulawinek, M., and Kozubek, A. (2008). "An improved colorimetric method for the determination of alkylresorcinols in cereals and whole-grain cereal products," Journal of Food Composition and Analysis 21(5), 428-434. DOI:10.1016/j.jfca.2008.04.002

Geerkens, C. H., Matejka, A. E., Carle, R., and Schweiggert, R. M. (2015). "Development and validation of an HPLC method for the determination of alk(en)ylresorcinols using rapid ultrasound-assisted extraction of mango peels and 
rye grains," Food Chemistry 169(7), 261-269. DOI:10.1016/j.foodchem.2014.08.001

Geerkens, C. H., Miller-Rostek, P., Matejka, A. E., Nene, S., Kammerer, D. R., Carle, R., and Schweiggert, R. M. (2015). "Influence of cultivar, ripeness, blanching, drying, irradiation, and pectin recovery on alk(en)ylresorcinols in mango peels," European Food Research and Technology 240(6), 1235-1245. DOI:10.1007/s00217015-2426-X

Gunenc, A., Hadinezhad, M., Tamburic-Ilincic, L., Mayer, P. M., and Hosseinian, F. (2013). "Effects of region and cultivar on alkylresorcinols content and composition in wheat bran and their antioxidant activity," Journal of Cereal Science 57(3), 405410. DOI:10.1016/j.jcs.2013.01.003

Hoogenboom, R., Wilms, T. F. A., Erdmenger, T., and Schubert, U. S. (2009). "Microwave-assisted chemistry: A closer look at heating efficiency," Australian Journal of Chemistry 62(3), 236-243. DOI:10.1071/CH08503

Jacobs, P. J., Hemdane, S., Delcour, J. A., and Courtin, C. M. (2016). "Dry heat treatment affects wheat bran surface properties and hydration kinetics," Food Chemistry 203, 513. DOI:10.1016/j.foodchem.2016.02.062

Kozubek, A., and Tyman, J. H. P. (1999). "Resorcinolic lipids, the natural nonisoprenoid phenolic amphiphiles and their biological activity," Cheminform 30(12), 1-25. DOI:10.1021/cr970464o

Landberg, R., Linko, A. M., Kamal-Eldin, A., Vessby, B., Adlercreutz, H., and Aman, P. (2006). "Human plasma kinetics and relative bioavailability of alkylresorcinols after intake of rye bran," Journal of Nutrition 136(11), 2760-2765.

Li, W., Pickard, M. D., and Beta, T. (2007). "Effect of thermal processing on antioxidant properties of purple wheat bran," Food Chemistry 104 (3), 1080-1086.

DOI:10.1016/j.foodchem.2007.01.024

Majewski, Ł., and Cunha, A.G. (2018). "Evaluation of suitability of wheat bran as a natural filler in polymer processing," Bioresources 13(3), 7037-7052.

DOI:10.15376/biores.13.3.7037-7052

Oladiran, D. A., Emmambux M. N., and de Kock, H. L. (2018). "Extrusion cooking of cassava-soy flour with $200 \mathrm{~g} / \mathrm{kg}$ wheat bran promotes slower oral processing during consumption of the instant porridge and higher derived satiety," LWT 97, 778-786. DOI:10.1016/j.lwt.2018.07.068

Podio, N. S., Baroni, M. V., Perez, G. T., and Wunderlin, D. A. (2019). "Assessment of bioactive compounds and their in vitro bioaccessibility in whole-wheat flour pasta," Food Chemistry 293, 408-417. DOI:10.1016/j.foodchem.2019.04.117

Randhir, R., Kwon, Y. I., and Shetty, K. (2008). "Effect of thermal processing on phenolics, antioxidant activity and health-relevant functionality of select grain sprouts and seedlings," Innovative Food Science and Emerging Technologies 9(3), 355-364. DOI:10.1016/j.ifset.2007.10.004

Rikard, L., Afaf, K. E., Marjatta, S. M., Xavier, R., and Per, Å. (2008). "Localization of alkylresorcinols in wheat, rye and barley kernels," Journal of Cereal Science 48(2), 401-406. DOI:10.1016/j.jcs.2007.09.013

Rose, D. J., Ogden, L. V., Dunn, M. L., and Pike, O. A. (2008). "Enhanced lipid stability in whole wheat flour by lipase inactivation and antioxidant retention," Cereal Chemistry 85(2), 218-223. DOI:10.1094/CCHEM-85-2-0218

Srivastava, A. K., Sudha, M. L., Baskaran, V., and Leelavathi, K. (2007). "Studies on heat stabilized wheat germ and its influence on rheological characteristics of dough," European Food Research and Technology 224(3), 365-372. DOI:10.1007/s00217- 
006-0317-x

Sudha, M. L., Srivastava, A. K., and Leelavathi, K. (2007). "Studies on pasting and structural characteristics of thermally treated wheat germ," European Food Research and Technology 225(3-4), 351-357. DOI: 10.1007/s00217-006-0422-x

Vadivambal, R., and Jayas, D. S. (2007). "Changes in quality of microwave-treated agricultural products--A review," Biosystems Engineering 98(1), 1-16. DOI:10.1016/j.biosystemseng.2007.06.006

Wang, P., Hou, C., Zhao, X., Tian, M., Gu, Z., and Yang, R. (2019). "Molecular characterization of water-extractable arabinoxylan from wheat bran and its effect on the heat-induced polymerization of gluten and steamed bread quality," Food Hydrocolloids 87, 570-581. DOI:10.1016/j.foodhyd.2018.08.049

Article submitted: November 1, 2019; Peer review completed: December 31, 2019; Revised version received: January 2, 2020; Accepted: January 6, 2020; Published: January 13, 2020.

DOI: $10.15376 /$ biores.15.1.1500-1509 